\title{
The role of catastrophizing in vulvovaginal pain: Impact on pain and partner responses over time
}

\section{Ida K. Flink ${ }^{1}$ (D) | Linnéa Engman ${ }^{1}$ | Johanna Thomtén ${ }^{2}$ | Steven J. Linton ${ }^{1}$}

${ }^{1}$ Center for Health and Medical Psychology (CHAMP), School of Law, Psychology, and Social Work, Örebro University, Örebro, Sweden

${ }^{2}$ Department of Psychology, Mid Sweden University, Östersund, Sweden

\section{Correspondence}

Ida K. Flink, School of Law, Psychology, and Social Work, Örebro University, Örebro, Sweden.

Email: ida.flink@oru.se
The aim of this prospective study was to explore the role of catastrophizing in vulvovaginal pain. The study entails two research questions. The first question was whether catastrophizing predicts the occurrence of vulvovaginal pain. The second question focused on exploring the links between catastrophizing, partner responses and pain. Five hundred and ten women filled out questionnaires at two measurement points, 10 months apart. The women were divided into three groups based on levels of catastrophizing. To study the first research question, the groups were used as predictors of pain at follow-up. To study the second research question, the groups were compared regarding their perceived solicitous partner responses. Subsequently, two mediation models were tested to explore whether catastrophizing mediated the relationship between solicitous partner responses and pain, crosssectionally and longitudinally. Baseline levels of catastrophizing distinguished between pain and no pain 10 months later. High catastrophizers reported higher levels of solicitous partner responses than low catastrophizers. The mediation analyses revealed catastrophizing as a significant mediator between solicitous partner responses and pain, cross-sectionally and over time. The findings support catastrophizing as being an important factor in women with vulvovaginal pain, cross-sectionally and over time. 


\section{1 | INTRODUCTION}

Vulvovaginal pain and other types of genital pain are strikingly common, with prevalence rates between $8 \%$ and $21 \%$ in premenopausal women, depending on diagnosis (e.g., Bachmann et al., 2006; Harlow et al., 2014). Women suffering from these problems report that the pain affects their engagement in daily activities (Masheb, Brondolo, \& Kerns, 2002), sexual activities, and sexual satisfaction (Smith \& Pukall, 2011), as well as relations with their partners (Ayling \& Ussher, 2008). For instance, the pain is not only linked to distress in the women themselves, but also to distress in their partners (Jodoin et al., 2008). The pain is further associated with overall consequences such as a lower quality of life (Arnold, Bachmann, Kelly, Rosen, \& Rhoads, 2006) and suicidal ideation (Gordon, Panahian-Jand, McComb, Melegari, \& Sharp, 2003). Despite these severe consequences, not least concerning relational aspects, there has been sparse research focusing on vulvovaginal pain from a pain research perspective (for a discussion, see Thomtén \& Linton, 2014). In particular, there are few longitudinal studies looking at the impact of psychological factors on pain and relational aspects over time. To understand how psychological factors influence further development of pain and long-term consequences, longitudinal data are a prerequisite. This prospective study will explore the role of catastrophizing in vulvovaginal pain, focusing on its impact on pain and relational aspects over a 10-month period. The study focuses on two separate research questions, of which the background will be described separately.

\section{1 | Research question I. The link between catastrophizing and vulvovaginal pain}

In other pain populations, catastrophizing has emerged as one of the most influential psychological factors determining pain outcomes (e.g., Keefe, Rumble, Scipio, Giordano, \& Perri, 2004; Leeuw et al., 2007; Leung, 2012; Quartana, Campbell, \& Edwards, 2009). The link between catastrophizing and pain has been established cross-sectionally as well as prospectively (for reviews, see Leung, 2012; Quartana et al., 2009; Sullivan et al., 2001) . Catastrophizing is commonly described as "an exaggerated negative mental set brought to bear during actual or anticipated pain experience" (Sullivan et al., 2001), and has been associated with a number of negative consequences such as prolonged problems and increased disability in various pain conditions (for reviews, see Keefe et al., 2004; Sullivan, et al., 2001). Taken together, catastrophizing is a well-known predominant risk factor in a wide range of pain populations.

Also in the area of vulvovaginal pain, there is growing evidence that catastrophizing plays a central role. For instance, women with vulvovaginal pain are reporting higher levels of catastrophizing than pain-free women (Alappattu et al., 2015; Anderson, Rosen, Price, \& Bergeron, 2016; Borg, Peters, Schultz, \& de Jong, 2012; Lemieux, Bergeron, Steben, \& Lambert, 2013; Thomtén \& Karlsson, 2014). There is also evidence that catastrophizing may predict pain intensity during vaginal intercourse (Desrochers, Bergeron, Khalifé, Dupuis, \& Jodoin, 2009). However, most studies have used cross-sectional data focusing on associations at a single point in time. To our knowledge, only one prospective study has explored the relation between catastrophizing and pain over time (Davis, Bergeron, Sadikaj, Corsini-Munt, \& Steben, 2015; Davis, Bergeron, Bois, et al., 2015). In that study, contrary to what is found in other pain populations, catastrophizing did not predict changes in pain intensity over a 2-year period in a sample of women with provoked vestibulodynia. In order to advance our understanding of the role of catastrophizing in vulvovaginal pain, the link between catastrophizing and pain needs to be further examined in longitudinal studies.

To this end, the first research question is whether pain catastrophizing at baseline predicts the occurrence of vulvovaginal pain 10 months later. We hypothesize that catastrophizing is a significant predictor of pain, in line with findings from other pain populations.

\section{2 | Research question II. The link between catastrophizing and partner responses: testing the Communal Coping Model in women with vulvovaginal pain}

As women with vulvovaginal pain frequently experience pain during vaginal intercourse, intimacy and the relation to the partner is often affected. One relational aspect which has received attention in other pain populations is how the 
partner responds to expressions of pain. Both soliciting responses, where the partner reacts with extensive sympathy and reinforces pain behavior, and punishing responses, where the partner is critical and questioning, have been associated with higher pain intensity in other pain populations (Boothby, Thorn, Overduin, \& Ward, 2004; Buenaver, Edwards, \& Haythornthwaite, 2007). In women with vulvovaginal pain, soliciting partner responses have been linked to higher pain intensity, whereas the findings with regard to punishing responses are mixed (Davis, Bergeron, Sadikaj et al., 2015; Davis, Bergeron, Bois, et al., 2015; Rosen, Bergeron, Leclerc, Lambert, \& Steben, 2010). As relational aspects are highly relevant in vulvovaginal pain, there is a need for further knowledge about the role of partner responses.

One theoretical model which incorporates relational aspects and partner responses as well as catastrophizing is the Communal Coping Model (CCM; Sullivan, 2012). According to the CCM, people who catastrophize might exaggerate their pain expressions in order to maximize support from people in their close surrounding (Sullivan, 2012). In this way, catastrophizing fulfills a communicative function and enables the person to cope with the pain by receiving soliciting responses as the result of pain behavior. Although there is no large evidence base supporting this model, catastrophizing has indeed been linked to higher levels of soliciting partner responses during shorter episodes of pain, and lower levels of soliciting responses when the pain persists (Buenaver et al., 2007). This is in line with the hypothesis in the CCM, suggesting that catastrophizing may initially maximize social support, but that the support decreases over a longer period of time. Partial support for the CCM was found in a study of women with provoked vulvodynia where catastrophizing mediated the link between solicitous partner responses and pain (Rosen, Bergeron, Lambert, \& Steben, 2013). However, they used a cross-sectional design, and it is unclear whether the same pattern would emerge over time.

The second research question is to explore the links between catastrophizing, partner responses and pain, crosssectionally and longitudinally using the CCM as the theoretical framework. More specifically, we are posing the following questions:

1. Do women with high levels of catastrophizing differ from women with lower levels in their perception of how their partner responds to expressions of pain? In line with the CCM, we hypothesize that women with high scores on catastrophizing will report higher levels of solicitous partner responses than women with low levels of catastrophizing. We do not have any hypotheses regarding perceived punishing responses, as the findings in previous studies have been mixed. Thus, we will look at the data exploratorily with regard to this aspect.

2. Does catastrophizing mediate the relation between perceived solicitous partner responses at baseline and ratings of pain intensity 10 months later? In line with the CCM as well as an earlier cross-sectional study (Rosen et al., 2013), we hypothesize that catastrophizing will be a significant mediator of the link between solicitous partner responses and pain.

\section{2 | METHODS}

\section{1 | Design}

This study used a subsample from a larger data set described elsewhere (Sex and Pain, SAP; Flink, Thomtén, Engman, Hedström, \& Linton, 2015). The original study included women with and without vulvovaginal pain who filled out self-report inventories at three measurement points. In this study, we used data from the first and the third measurement point, which were administered 10 months apart.

\section{2 | Recruitment}

Women between 18 and 35 years old were recruited at two middle-sized universities in Sweden. All women in 66 classes were invited to remain in their classrooms after lectures to receive information about the study. Once 
agreeing to participate, the woman filled out an informed consent, picked a questionnaire from an open box in the classroom, completed the questionnaire, and put it in a blank envelope in a closed box in the classroom. If she preferred to fill out the questionnaire at home, she was allowed to bring it and hand it in later, in a closed box at the research center (CHAMP). Coffee coupons were provided as compensation. All questionnaires were coded. The informed consent form, which contained information about the woman's addresses, was handled and stored separately. The codes were used for linking the first and the last questionnaires to each other. Five and 10 months later, identical questionnaires were sent to the woman's home address, together with written information about the study and pre-paid envelopes for returning the questionnaires. Two and 4 weeks after sending out the questionnaires, e-mail reminders were sent out to non-responders. The women were once again reminded of that all questionnaires were coded, and only the codes were used for linking the first and the last questionnaires to each other. Responders received cinema tickets as compensation after returning the questionnaire. The study was ethically approved by the Regional Ethical Review Board in Uppsala, Sweden (D Number 2014-407).

\section{3 | Participants}

In total, 1,034 women completed the questionnaire at the first measurement point. Twelve women were excluded because of a lack of informed consent. Out of the remaining 1,022 women, 85 women were excluded due to not being sexually active (derived from the question "Are you sexually active? By yourself or with a partner") and additionally 22 were excluded because of too many missing values on the measures used in the study ( $>20 \%$ missing values/subscale). Out of the remaining 915 women, 510 (56\%) answered the questionnaire at the second measurement point 10 months later. These 510 women constitute the sample of this study, see Table 1 for baseline characteristics.

An analysis of non-responders between responders at time point 1 only $(N=405)$ and responders answering at both time points $(N=510)$ was performed on the variables age, pain catastrophizing, experienced partner responses

TABLE 1 Demographic data of the sample at baseline $(N=510)$

\begin{tabular}{|c|c|}
\hline \multicolumn{2}{|l|}{ Age (years) } \\
\hline Mean (SD) & $23.40(3.4)$ \\
\hline \multicolumn{2}{|l|}{ Relationship } \\
\hline Yes $(n, \%)$ & $331(65 \%)$ \\
\hline No $(n, \%)$ & $164(32 \%)$ \\
\hline Other ( $n, \%)$ & $14(3 \%)$ \\
\hline \multicolumn{2}{|c|}{ Relationship length (years) } \\
\hline Mean (SD) & $3.10(2.6)$ \\
\hline \multicolumn{2}{|l|}{ Gender of partner } \\
\hline Male (n, \%) & $331(96.5 \%)$ \\
\hline Female ( $n, \%)$ & $10(3 \%)$ \\
\hline Other $(n, \%)$ & $2(0.5 \%)$ \\
\hline \multicolumn{2}{|l|}{ Children } \\
\hline Yes $(n, \%)$ & $34(7 \%)$ \\
\hline No $(n, \%)$ & $473(93 \%)$ \\
\hline \multicolumn{2}{|c|}{ Recurring vulvovaginal pain ( $\geq 6$ months) } \\
\hline Yes $(n, \%)$ & $161(32 \%)$ \\
\hline No $(n, \%)$ & $349(68 \%)$ \\
\hline \multicolumn{2}{|c|}{ Pain intensity in the pain sample $(0-10)$} \\
\hline Mean (SD) & 3.63 (1.98) \\
\hline
\end{tabular}


(solicitous and punishing), and pain intensity, showing no significant differences between the groups $(t(915)=-.64$, $p=.52 ; t(915)=-1.34, p=.18 ; t(265)=1.27, p=.20 ; t(282)=.89, p=.37 ; t(293)=-.36, p=.72)$. At time point 2 , 145 (28\%) women reported recurring vulvovaginal pain during the last 6 months, while 365 women (72\%) reported no pain.

\section{4 | Measures}

Swedish versions of all measures were used.

\subsection{1 | Demographics}

Demographic data regarding age, relationship status, and children were collected, based on questions used in earlier studies on this population (Thomtén \& Karlsson, 2014).

\subsection{2 | Vulvovaginal pain}

The occurrence of vulvovaginal pain was assessed with the question "Have you experienced recurrent pain during sexual intercourse/touch/contact with the vulva during the last 6 months?" (Yes/No). Pain intensity was assessed using the question "How would you rate the level of pain during the last three months?". Answers were given on an 11-point numerical rating scale (NRS) scale ranging from 0 ("No pain") to 10 ("Worst pain imaginable"). For the women that did not experience pain, the pain intensity was reported as 0 .

\subsection{3 | Pain catastrophizing}

Pain-related catastrophizing was assessed with the Pain Catastrophizing Scale (PCS) (Sullivan, Bishop, \& Pivik, 1995). The PCS contains 13 thoughts and feelings that people may report when in pain (e.g., "I keep thinking of other painful events";" I feel I can't go on"). Answers are given on a five-point scale ( 0 = Not at all; 4 = All the time), which indicates to what extent they experience each thought and feeling when in pain. The PCS has shown satisfactory psychometric properties (Sullivan et al., 1995). In this study, the total score of the PCS was used, and the internal consistency was good (Cronbach's $\alpha=.91$ ).

\subsection{4 | Partner responses}

Partner responses to pain were assessed with two subscales of the West-Haven Multidimensional Pain Inventory (MPI) (Kerns, Turk, \& Rudy, 1985). The MPI includes three types of partner responses: solicitous, punishing, and distracting responses. This study only included the solicitous (e.g., "Asks me how he/she can help") and punishing subscale (e.g., "Ignores me"). Answers are given on a seven-point scale, indicting how often the woman experiences the spouse to respond in specific ways when expressing pain $(0=$ never; $6=$ very often). Due to an uneven number of questions on each subscale, the subscales in this study were standardized so that the total score of each subscale ranged from 0 to 6 . Only women reporting recurring vulvovaginal pain in combination with being in a relationship answered on this variable. The subscales of the MPI have shown adequate psychometric properties (Kerns et al., 1985). In this study, the internal consistency was good (Cronbach's $\alpha=.80$ vs. .74).

\section{5 | Data analyses}

The analyses were performed using the IBM Statistical Package of Social Sciences (SPSS) 24.0. The individual's mean score of the subscale was used for replacing missing items, allowing one missing value/subscale, or maximum $20 \%$. 
First, the data were summarized and inspected through descriptive and correlational statistics. Next, the women were divided into groups based on their sum score on the PCS at time point 1. According to recommendations (Sullivan, 2009), the following labels were used: low (0-19), moderate (20-29), and high (30 and above) (Sullivan, 2009).

To predict pain after 10 months based on level of catastrophizing at time point 1 , a logistic regression analysis was performed, where the probability of reporting pain after 10 months was expressed by odds ratio in the three groups with different levels of catastrophizing. The group with low levels of catastrophizing was used as the comparison group.

Regarding the second research question, the groups of women with low, moderate, and high levels of catastrophizing were compared on their perceived solicitous partner responses through analysis of variance (ANOVA). Hochberg's GT2 was chosen as the post hoc analysis due to unequal sizes of the groups. Subsequently, two simple mediation models were explored where pain catastrophizing was proposed to mediate the relationship between solicitous partner responses and pain, cross-sectionally and over time. Two different approaches were used: the stepwise approach by Baron and Kenny (1986) to examine the basic requirements of mediation analysis, and Preacher and Hayes (2008) method of examining the significance of the indirect effect by bootstrapping biased controlled confidence-intervals of the indirect effects ( $n=5,000$ bootstrap resamples). Effect sizes of the indirect effects are expressed in $k^{2}$ which is the ratio of the observed indirect effect to the maximum possible indirect effect. The recommendations for interpretation of $k^{2}$ are: small .01; medium .09; large .25 (Preacher \& Kelley, 2011). The level of significance was set to $p<.05$ in all analyses.

\section{3 | RESULTS}

First, descriptive statistics and correlations between the variables used in the studies were examined. As displayed in Table 2, there was a small positive correlation between baseline ratings of pain catastrophizing and the women's perceived solicitous partner responses at time point 1 (baseline) $(r=.21, p<.05)$ as well as with reported pain intensity at time point $2(r=.19, p<.05)$. There was a small negative correlation between perceived punishing and solicitous partner response at time point $1(r=-.23, p<.01)$. There was no significant correlation between pain catastrophizing and the women's perceived punishing partner responses $(r=.11, \mathrm{NS})$.

\section{1 | Research question I. The link between catastrophizing and vulvovaginal pain over time}

To explore whether baseline catastrophizing may predict the occurrence of pain at time point 2, the women were divided into three groups based on their scores on the PCS: low catastrophizing $(N=316)$, medium catastrophizing $(N=125)$, and high catastrophizing $(N=69)$.

TAB LE 2 Descriptive statistics and correlations between the main variables in the studies

\begin{tabular}{|c|c|c|c|c|c|c|}
\hline & $N$ & Range & $M(S D)$ & 2 & 3 & 4 \\
\hline 1. Pain catastrophizing (PCS, $t 1)$ & 510 & $0-52$ & $17.37(9.91)$ & $.21^{*}$ & .11 & $.19^{*}$ \\
\hline $\begin{array}{l}\text { 2. Solicitous partner responses } \\
(\mathrm{MPI}-\mathrm{S}, t 1)\end{array}$ & 148 & $0-6$ & $3.21(1.42)$ & - & $-.23^{* *}$ & .04 \\
\hline $\begin{array}{l}\text { 3. Punishing partner responses } \\
(\mathrm{MPI}-\mathrm{P}, \mathrm{t} 1)\end{array}$ & 148 & $0-6$ & $0.56(0.81)$ & & - & -.007 \\
\hline 4. Pain intensity ${ }^{a}(N R S, t 2)$ & 148 & $0-10$ & $2.32(2.72)$ & & & - \\
\hline
\end{tabular}

PCS, Pain Catastrophizing scale; MPI-S and MPI-P, West-Haven Multidimensional Pain Inventory; $t 1$ and $t 2$, time point 1 and 2. ${ }^{\text {a }}$ Participants responding to the PCS and the MPI at $t 1$. ${ }^{*} p<.05 ;{ }^{* *} p<.01 ;{ }^{* * *} p<.001$. 
A logistic regression analysis was conducted to explore the association between the groups and the occurrence of pain at time point 2. As displayed in Table 3, the logistic regression model was significant, indicating that the level of catastrophizing at baseline reliably distinguished between pain and no pain 10 months later $\left(\chi^{2}=13.26, p<.001\right.$, $d f=2$ ). Women in the medium catastrophizing group were 1.68 times more likely to report pain at time point 2 than women in the low catastrophizing group, while women in the high catastrophizing group were 2.56 times more likely to report pain at time point 2 compared to the low catastrophizing group.

\subsection{Research question II. The link between catastrophizing and relational aspects}

To examine the link between catastrophizing and perceived solicitous partner responses, the three groups of women with different levels of catastrophizing were compared on their perceived solicitous partner responses at time point 1. As displayed in Table 4, the analysis of variance showed that there were significant differences between the groups $(F(2,145)=3.17, p<.05)$ with the post hoc analysis revealing that the high catastrophizing group reported significantly higher levels of solicitous partner responses than the low catastrophizing group (mean difference $=0.73$, $p<.05)$. In other words, the women reporting high levels of catastrophizing also reported higher levels of solicitous partner responses, compared to the women with low levels of catastrophizing.

To investigate whether pain catastrophizing mediated the relationship between solicitous partner responses and pain intensity, two mediation models were tested-one cross-sectionally with all variables at time point 1 and one longitudinally with pain intensity measured at time point 2 . Table 5 displays the regression results for the two mediation models.

The simple mediation analysis at time point 1 showed a decrease in the total effect $(c, \beta=.16, p=.057)$ compared to the direct effect $\left(c^{\prime}, \beta=.09\right.$, NS) for solicitous partner responses on pain intensity when catastrophizing was added as a mediator in the model, see Figure 1a. The bootstrapped indirect effect for pain catastrophizing as a mediator

TAB LE 3 Logistic regression predicting pain (yes/no) at time point 2 based on level of catastrophizing at time point $1(N=510)$

\begin{tabular}{|c|c|c|c|c|c|}
\hline & $R^{2}$ & $\beta$ & SE & Odds ratio & $p$ \\
\hline \multicolumn{6}{|l|}{ Model } \\
\hline Constant & & -1.20 & .13 & 0.30 & $<.001$ \\
\hline Medium catastrophizing & & 0.52 & .23 & 1.68 & $<.05$ \\
\hline High catastrophizing & & 0.94 & .28 & 2.56 & $<.001$ \\
\hline (base $=$ low catastrophizing) & .04 & & & & \\
\hline
\end{tabular}

Notes: Levels of catastrophizing based on scorings on the PCS, Pain Catastrophizing Scale, at time point 1; low catastrophizing $=0-19$, medium catastrophizing $=20-29$, high catastrophizing $\geq 30$. The low catastrophizing group was used as the comparison group.

TAB LE 4 Differences on experienced solicitous partner responses in the groups of women with different levels of catastrophizing $(N=148)$

\begin{tabular}{|lccc|} 
& N & Mean & SD \\
\hline Low catastrophizing & 77 & 3.01 & 1.41 \\
\hline Medium catastrophizing & 38 & 3.15 & 1.38 \\
\hline High catastrophizing & 33 & 3.74 & 1.39 \\
\hline Total sample & 148 & 3.21 & 1.42 \\
\hline
\end{tabular}

Notes: Levels of catastrophizing based on scorings on the PCS, Pain Catastrophizing Scale, at time point 1; low catastrophizing $=0-19$, medium catastrophizing $=20-29$, high catastrophizing $\geq 30$. Solicitous partner responses measured by the MPI-S, West-Haven Multidimensional Pain Inventory, scores ranging between 0-6. 
TABLE 5 Regression results for the mediation analyses of experienced solicitous partner responses on pain intensity by pain catastrophizing, cross-sectionally and over time

\begin{tabular}{|c|c|c|c|c|c|}
\hline Model & $R^{2}$ & $\boldsymbol{\beta}$ & SE & $p$ & $\mathrm{Cl}(95 \%)$ \\
\hline \multicolumn{6}{|l|}{ Cross-sectionally $(N=146)$} \\
\hline \multicolumn{6}{|l|}{ Model without mediator } \\
\hline Solicitous response $\rightarrow$ Pain $(c)$ & & .158 & .082 & $.057, \mathrm{NS}$ & -.005 to .049 \\
\hline$R_{Y, X}^{2}$ & .03 & & & & \\
\hline \multicolumn{6}{|l|}{ Model with mediator } \\
\hline Solicitous response $\rightarrow$ Catastrophizing (a) & & .222 & .087 & $<.05$ & .049 to .394 \\
\hline Catastrophizing $\rightarrow$ Pain $(b)$ & & .313 & .074 & $<.001$ & .166 to .460 \\
\hline Solicitous response $\rightarrow$ Pain $\left(c^{\prime}\right)$ & & .088 & .079 & $.269, \mathrm{NS}$ & -.069 to .245 \\
\hline Indirect effect $(a \times b)$ & & .069 & .035 & & .011 to .152 \\
\hline$R_{M, X}^{2}$ & .04 & & & & \\
\hline$R_{Y, M X}^{2}$ & .13 & & & & \\
\hline \multicolumn{6}{|l|}{ Over time $(N=148)$} \\
\hline \multicolumn{6}{|l|}{ Model without mediator } \\
\hline Solicitous response $\rightarrow$ Pain $(c)$ & & .052 & .110 & $.635, \mathrm{NS}$ & -.165 to .270 \\
\hline$R_{Y, X}^{2}$ & .002 & & & & \\
\hline \multicolumn{6}{|l|}{ Model with mediator } \\
\hline Solicitous response $\rightarrow$ Catastrophizing $(a)$ & & .227 & .090 & $<.05$ & .049 to. 406 \\
\hline Catastrophizing $\rightarrow$ Pain $(b)$ & & .226 & .100 & $<.05$ & .030 to. 423 \\
\hline Solicitous response $\rightarrow$ Pain $\left(c^{\prime}\right)$ & & .001 & .111 & $.994, \mathrm{NS}$ & -.218 to .220 \\
\hline Indirect effect $(a \times b)$ & & .052 & .032 & & .007 to .140 \\
\hline$R_{M, X}^{2}$ & .04 & & & & \\
\hline$R_{Y, M X}^{2}$ & .04 & & & & \\
\hline
\end{tabular}

$R_{Y, X}^{2}$, proportion of the variance in the pain variable $(Y)$ explained by solicitous partner responses $(X) ; R_{M, X}^{2}$, proportion of the variance in the catastrophizing variable $(M)$ explained by solicitous partner responses $(X) ; R_{Y, M X}^{2}$, is the proportion of the variance in the pain variable $(Y)$ explained by catastrophizing $(M)$ and solicitous partner responses $(X)$; NS, non-significant.

The $95 \% \mathrm{Cl}$ for $a \times b$ is obtained by the bias-corrected bootstrap with 5,000 resamples. Standardized regression weights $a$, $b, c$, and $c^{\prime}$ are illustrated in Figure 1.

was significant $(a \times b, \beta=.07,95 \% \mathrm{BCl}[.011, .152])$, excluding zero in the confidence interval, with a small effect size $\left(k^{2}=.07(95 \% \mathrm{BCl}[.013, .146])\right.$.

Over time, there was also a decrease in the total effect ( $c, \beta=.05$, NS) compared to the direct effect $\left(c^{\prime}, \beta=.001\right.$, NS) when catastrophizing was proposed to mediate the relationship between solicitous partner responses and pain intensity at time point 2 , see Figure $1 \mathrm{~b}$. There was a significant indirect effect of catastrophizing as a mediator over time, excluding zero in the bootstrapped confidence interval $(a \times b, \beta=.052,95 \% \mathrm{BCl}[.007, .140])$, which represents a small indirect effect $\left(k^{2}=.04,95 \% \mathrm{BCl}[.006, .103]\right)$.

\section{DISCUSSION}

This study explored the role of catastrophizing in women with vulvovaginal pain over time. With regard to the first research question, baseline levels of catastrophizing were found to predict the occurrence of vulvovaginal pain 10 months later, in line with our hypothesis. The second research question focused on exploring the links between 


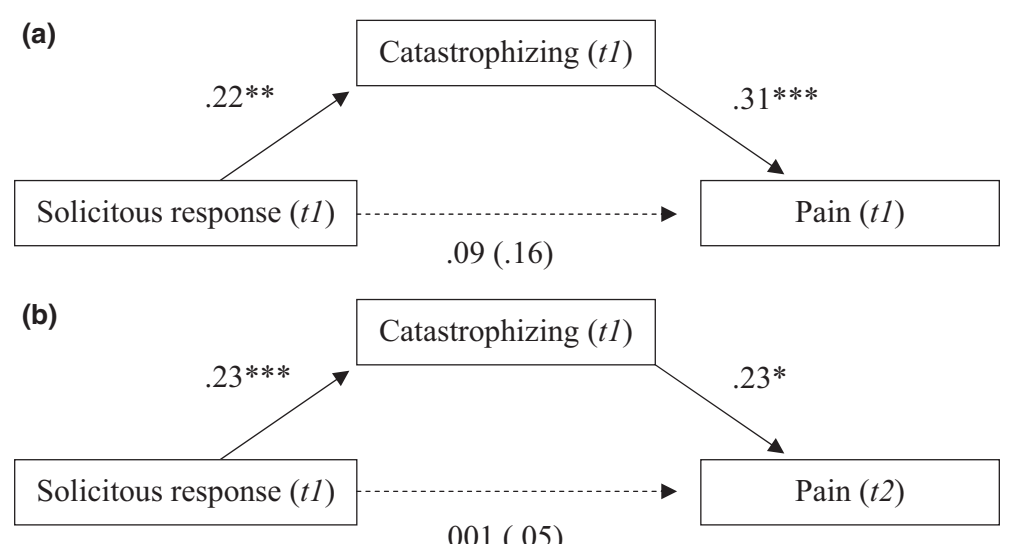

$.001(.05)$

FIGURE 1 Standardized regression coefficients for the relationship between perceived solicitous partner responses and pain intensity as mediated by pain catastrophizing, cross-sectionally (a) and over time (b). Standardized regression coefficient of the total effect between solicitous responses and pain is in parentheses. $t_{1}$ and $t_{2}$, time point 1 and 2

catastrophizing, partner responses and pain, cross-sectionally and longitudinally in the group of women who already suffered from pain at baseline, using the Communal Coping Model of catastrophizing (CCM) as the theoretical framework. In line with the CCM as well as with our hypothesis, women with high levels of catastrophizing reported significantly higher levels of solicitous partner responses than women scoring low on catastrophizing. Regarding punishing responses, there was no significant correlation with catastrophizing, and therefore this association was not explored further. When scrutinizing the links between catastrophizing, partner responses and pain, the mediation analyses revealed catastrophizing as a significant mediator of the relation between solicitous partner responses and pain, cross-sectionally as well as longitudinally. This provided support for the CCM and was in line with our hypotheses.

The first research question focused on catastrophizing as a potential predictor of pain 10 months later-a question where the answer may seem obvious when looking at other pain populations where catastrophizing is commonly referred to as one of the most influential predictors of pain (Leung, 2012; Quartana et al., 2009; Sullivan et al., 2001). In the area of vulvovaginal pain, however, the only study that we are aware of looking into this relationship over time, found that catastrophizing did not predict changes in pain intensity 2 years later in a sample of women with provoked vestibulodynia (Davis, Bergeron, Sadikaj et al., 2015; Davis, Bergeron, Bois, et al., 2015). The contradicting findings may have several explanations. First, our study looked at a non-clinical sample, including both women with and without vulvovaginal pain broadly defined and not in terms of clinical diagnoses, with the occurrence of pain as the outcome. This differs from the study by Davis and colleagues, who focused on changes in pain intensity in women with a specific diagnosis. In one sense, these are two separate questions to focus on the role of catastrophizing in the development of pain, a non-clinical sample might be preferable, whereas a clinical sample provides an opportunity to look at changes in pain over time. Moreover, our second measurement point was 10 months later, as opposed to 2 years, which may have facilitated revealing significant effects on the outcome. Another factor may be the size of the association where the correlation we report between baseline catastrophizing and pain intensity at follow-up was small $(r=.19)$. Nevertheless, the current findings contribute to the literature on psychological factors in vulvovaginal pain by pointing out catastrophizing as a significant risk factor for pain, similar to what is found in other pain populations.

Regarding our second research question, the results are in line with the CCM as well as with earlier studies investigating the links between partner responses, catastrophizing and pain. In fact, we replicate and extend the findings by Rosen et al. (2013), who found that catastrophizing mediated the relation between perceived solicitous partner responses and pain cross-sectionally, if only with small effects. It is worth noting that before catastrophizing was included as a mediator, the effects of solicitous responses on pain were not significant, neither in the study by Rosen 
et al. (2013), nor in our study. Nevertheless, when including the mediator, significant indirect effects were observed both concurrently and over time, providing support for catastrophizing as a mediator of the relation between solicitous partner responses and pain. Keeping the small effects in mind, the clinical significance of these results may be limited, although they are theoretically relevant, not least by providing support for the CCM.

The CCM suggests that catastrophizing may serve a communicative function through eliciting social support, for instance from a partner (Sullivan, 2012). While a number of studies in other pain populations have endorsed the CCM in confirming the link between catastrophizing and the social environment (e.g., Giardino, Jensen, Turner, Ehde, \& Cardenas, 2003; Keefe et al., 2003; Lackner \& Gurtman, 2004; Sullivan, Adams, \& Sullivan, 2004), the model has also received substantial criticism (Flink, Boersma, \& Linton, 2013; Severeijns, Vlaeyen, \& van den Hout, 2006), and has not entirely come through as a dominating framework for understanding catastrophizing. It might be that the CCM is most applicable for pain conditions where the interpersonal context is of greater importance, for instance when the pain occurs in intimate situations. While the CCM is appropriate here given the social nature of the problem, it also has shortcomings. For instance, the CCM does not take into account the strong interrelation between catastrophizing and worry behavior (Flink et al., 2013). In the context of vulvovaginal pain, the worry might concern relational aspects such as losing the relationship. This may result in the woman engaging in behaviors to prevent feared consequences, instead of sincerely communicating her needs to the partner. Although our findings add to the emerging evidence for CCM as being a firm theoretical framework for explaining the links between catastrophizing, partner responses and vulvovaginal pain, there is a need for further developing other theoretical frameworks around catastrophizing to fully understand its nature.

While this study contributes to the existing literature, not least by being one of the first longitudinal studies on catastrophizing in vulvovaginal pain, the methodological shortcomings need to be taken into consideration. One drawback is that information is lacking about the women who chose not to participate in the study, inferring a possible selection bias. However, since this is not an epidemiological study where a representative sample is a prerequisite, the exploration of interrelations between psychological mechanisms should be less sensitive to specific characteristics of the sample. Another drawback is the assessment of perceived partner responses. An observable measure would have provided a more complete picture of the interpersonal processes involved. Yet, it is in fact plausible that the woman's experience of the response is critical, and not the response in itself, when examining its consequences on outcomes. Another shortcoming may be that only two possible partner responses were assessed, namely solicitous and punishing responses. Assessment of facilitative responses may also be of interest as these have been identified as more adaptive for pain outcomes in a sample with provoked vestibulodynia (Rosen, Bergeron, Glowacka, Delisle, \& Baxter, 2012). Future studies on this topic should preferably include measures of facilitative responses. The methodological choice of dividing the women into groups with different levels of catastrophizing may be pedagogically sound and allows comparison of the groups, for instance on levels of perceived partner responses. However, as it reduces the variability in the data, it may also have resulted in limited power, possibly leading to somewhat smaller effects.

Even though the effects in this study were relatively small, they nevertheless advance our understanding of the role of catastrophizing in vulvar pain. The result that baseline levels of catastrophizing predicted pain over time is not only of theoretical value, but may also point at the need for identifying and addressing catastrophizing early on, to counteract the development of chronic problems. Moreover, taking into consideration the interaction between perceived partner responses and catastrophizing, it may be of value to engage the partner in this work. However, further studies are needed to reveal effective methods to work with catastrophizing, partner responses, and pain. One potential avenue is to train the partner in validation, which in essence means to communicate empathy and understanding, at the same time as assisting the woman in approaching long-term goals (Fruzzetti \& Iverson, 2004), such as maintaining intimacy. Vulvovaginal pain has significant consequences by affecting sexual activities and satisfaction (Smith \& Pukall, 2011), and it is therefore essential to identify risk factors for persistent problems, as well as developing effective means for assisting women in coping with this pain early on. 


\section{REFERENCES}

Alappattu, M. J., George, S. Z., Robinson, M. E., Fillingim, R. B., Moawad, N., Lebrun, E. W., \& Bishop, M. D. (2015). Painful intercourse is significantly associated with evoked pain perception and cognitive aspects of pain in women with pelvic pain. Sexual Medicine, 3, 14-23.

Anderson, A. B., Rosen, N. O., Price, L., \& Bergeron, S. (2016). Associations between penetration cognitions, genital pain, and sexual well-being in women with provoked vestibulodynia. The Journal of Sexual Medicine, 13(3), 444-452.

Arnold, L. D., Bachmann, G. A., Kelly, S., Rosen, R., \& Rhoads, G. G. (2006). Vulvodynia: characteristics and associations with co-morbidities and quality of life. Obstetrics and Gynecology, 107(3), 617.

Ayling, K., \& Ussher, J. M. (2008). "If sex hurts, am I still a woman?" The subjective experience of vulvodynia in hetero-sexual women. Archives of Sexual Behavior, 37(2), 294-304.

Bachmann, G. A., Rosen, R., Arnold, L. D., Burd, I., Rhoads, G. G., Leiblum, S. R., \& Avis, N. (2006). Chronic vulvovaginal and gynecologic pain: Prevalence and characteristics in a self-reported survey. The Journal of Reproductive Medicine, 51(1), 3.

Baron, R. M., \& Kenny, D. A. (1986). The moderator-mediator variable distinction in social psychological research: Conceptual, strategic, and statistical considerations. Journal of Personality and Social Psychology, 51(6), 1173.

Boothby, J. L., Thorn, B. E., Overduin, L. Y., \& Ward, L. C. (2004). Catastrophizing and perceived partner responses to pain. Pain, 109(3), 500-506.

Borg, C., Peters, M. L., Schultz, W. W., \& de Jong, P. J. (2012). Vaginismus: Heightened harm avoidance and pain catastrophizing cognitions. The Journal of Sexual Medicine, 9(2), 558-567.

Buenaver, L. F., Edwards, R. R., \& Haythornthwaite, J. A. (2007). Pain-related catastrophizing and perceived social responses: Inter-relationships in the context of chronic pain. Pain, 127(3), 234-242.

Davis, S. N. P., Bergeron, S., Bois, K., Sadikaj, G., Binik, Y. M., \& Steben, M. (2015). A prospective 2-year examination of cognitive and behavioral correlates of provoked vestibulodynia outcomes. Clinical Journal of Pain, 31(4), 333-341.

Davis, S. N., Bergeron, S., Sadikaj, G., Corsini-Munt, S., \& Steben, M. (2015). Partner behavioral responses to pain mediate the relationship between partner pain cognitions and pain outcomes in women with provoked vestibulodynia. The Journal of Pain, 16(6), 549-557.

Desrochers, G., Bergeron, S., Khalifé, S., Dupuis, M. J., \& Jodoin, M. (2009). Fear avoidance and self-efficacy in relation to pain and sexual impairment in women with provoked vestibulodynia. The Clinical Journal of Pain, 25(6), 520-527.

Flink, I. K. L., Boersma, K., \& Linton, S. J. (2013). Pain catastrophizing as repetitive negative thinking: A development of the conceptualization. Cognitive Behaviour Therapy, 42(3), 215-223.

Flink, I. K., Thomtén, J., Engman, L., Hedström, S., \& Linton, S. J. (2015). Coping with painful sex: Development and initial validation of the CHAMP Sexual Pain Coping Scale. Scandinavian Journal of Pain, 9, 74-80.

Fruzzetti, A. E., \& Iverson, K. M. (2004). Mindfulness, acceptance, validation, and "individual" psychopathology in couples. In Hayes, Follette \& Linehan (Eds.), Mindfulness and acceptance: Expanding the cognitive-behavioral tradition (pp. 168-191). New York: The Guilford Press.

Giardino, N. D., Jensen, M. P., Turner, J. A., Ehde, D. M., \& Cardenas, D. D. (2003). Social environment moderates the association between catastrophizing and pain among persons with a spinal cord injury. Pain, 106(1), 19-25.

Gordon, A. S., Panahian-Jand, M., McComb, F., Melegari, C., \& Sharp, S. (2003). Characteristics of women with vulvar pain disorders: Responses to a web-based survey. Journal of Sex \& Marital Therapy, 29(Supp 1), 45-58.

Harlow, B. L., Kunitz, C. G., Nguyen, R. H., Rydell, S. A., Turner, R. M., \& MacLehose, R. F. (2014). Prevalence of symptoms consistent with a diagnosis of vulvodynia: Population-based estimates from 2 geographic regions. American Journal of Obstetrics and Gynecology, 210(1), 40.e1.

Jodoin, M., Bergeron, S., Khalifé, S., Dupuis, M. J., Desrochers, G., \& Leclerc, B. (2008). Male partners of women with provoked vestibulodynia: Attributions for pain and their implications for dyadic adjustment, sexual satisfaction, and psychological distress. The Journal of Sexual Medicine, 5(12), 2862-2870.

Keefe, F. J., Lipkus, I., Lefebvre, J. C., Hurwitz, H., Clipp, E., Smith, J., \& Porter, L. (2003). The social context of gastrointestinal cancer pain: a preliminary study examining the relation of patient pain catastrophizing to patient perceptions of social support and caregiver stress and negative responses. Pain, 103(1), 151-156.

Keefe, F. J., Rumble, M. E., Scipio, C. D., Giordano, L. A., \& Perri, L. M. (2004). Psychological aspects of persistent pain: Current state of the science. The Journal of Pain, 5(4), 195-211.

Kerns, R. D., Turk, D. C., \& Rudy, T. E. (1985). The West Haven-Yale Multidimensional Pain Inventory (WHYMPI). Pain, 23(4), 345-356.

Lackner, J. M., \& Gurtman, M. B. (2004). Pain catastrophizing and interpersonal problems: A circumplex analysis of the communal coping model. Pain, 110(3), 597-604.

Leeuw, M., Goossens, M. E., Linton, S. J., Crombez, G., Boersma, K., \& Vlaeyen, J. W. (2007). The fear-avoidance model of musculoskeletal pain: Current state of scientific evidence. Journal of Behavioral Medicine, 30(1), 77-94. 
Lemieux, A. J., Bergeron, S., Steben, M., \& Lambert, B. (2013). Do romantic partners' responses to entry dyspareunia affect women's experience of pain? The roles of catastrophizing and self-efficacy. The Journal of Sexual Medicine, 10(9), 2274-2284.

Leung, L. (2012). Pain catastrophizing: An updated review. Indian Journal of Psychological Medicine, 34(3), 204.

Masheb, R. M., Brondolo, E., \& Kerns, R. D. (2002). A multidimensional, case-control study of women with self-identified chronic vulvovaginal pain. Pain Medicine, 3(3), 253-259.

Preacher, K. J., \& Hayes, A. F. (2008). Asymptotic and resampling strategies for assessing and comparing indirect effects in multiple mediator models. Behavior Research Methods, 40(3), 879-891.

Preacher, K. J., \& Kelley, K. (2011). Effect sizes measures for mediation models: Quantitative strategies for communicating indirect effects. Psychological Methods, 16(2), 93-115.

Quartana, P. J., Campbell, C. M., \& Edwards, R. R. (2009). Pain catastrophizing: A critical review. Expert Review of Neurotherapeutics, 9(5), 745.

Rosen, N. O., Bergeron, S., Glowacka, M., Delisle, I., \& Baxter, M. L. (2012). Harmful or helpful: Perceived solicitous and facilitative partner responses are differentially associated with pain and sexual satisfaction in women with provoked vestibulodynia. The Journal of Sexual Medicine, 9(9), 2351-2360.

Rosen, N. O., Bergeron, S., Lambert, B., \& Steben, M. (2013). Provoked vestibulodynia: Mediators of the associations between partner responses, pain, and sexual satisfaction. Archives of Sexual Behavior, 42(1), 129-141.

Rosen, N. O., Bergeron, S., Leclerc, B., Lambert, B., \& Steben, M. (2010). Woman and partner-perceived partner responses predict pain and sexual satisfaction in provoked vestibulodynia (PVD) couples. The Journal of Sexual Medicine, 7(11), 3715-3724.

Rosen, C., Brown, J., Heiman, S., Leiblum, C., Meston, R., Shabsigh, D., ... D’Agostino, R. (2013). The Female Sexual Function Index (FSFI): A multidimensional self-report instrument for the assessment of female sexual function. Journal of Sex \& Marital Therapy, 26(2), 191-208.

Severeijns, R., Vlaeyen, J. W. S., \& van den Hout, M. A. (2006). Do we need a communal coping model of pain catastrophizing? An alternative explanation Pain, 11(1), 226-229.

Smith, K. B., \& Pukall, C. F. (2011). A systematic review of relationship adjustment and sexual satisfaction among women with provoked vestibulodynia. Journal of Sex Research, 48(2-3), 166-191.

Sullivan, M. J. L. (2009). The pain catastrophizing scale: User manual (pp. 1-36). Montreal: McGill University.

Sullivan, M. J. (2012). The communal coping model of pain catastrophising: Clinical and research implications. Canadian Psychology/Psychologie Canadienne, 53(1), 32.

Sullivan, M. J., Thorn, B., Haythornthwaite, J. A., Keefe, F., Martin, M., Bradley, L. A., \& Lefebvre, J. C. (2001). Theoretical perspectives on the relation between catastrophizing and pain. Clinical Journal of Pain, 17(1), 52-64.

Sullivan, M. J. L., Adams, H., \& Sullivan, M. E. (2004). Communicative dimensions of pain catastrophizing: Social cueing effects on pain behaviour and coping. Pain, 107(3), 220-226.

Sullivan, M. J., Bishop, S. R., \& Pivik, J. (1995). The pain catastrophizing scale: Development and validation. Psychological Assessment, 7(4), 524.

Thomtén, J., \& Karlsson, A. (2014). Psychological factors in genital pain: The role of fear-avoidance, pain catastrophizing and anxiety sensitivity among women living in Sweden. Scandinavian Journal of Pain, 5(3), 193-199.

Thomtén, J., \& Linton, S. J. (2014). When sex hurts: Female genital pain with sexual consequences deserves attention: A position paper. Scandinavian Journal of Pain, 5(3), 202-205.

How to cite this article: Flink IK, Engman L, Thomtén J, Linton SJ. The role of catastrophizing in vulvovaginal pain: Impact on pain and partner responses over time. J Appl Behav Res. 2017;22:e12093. https://doi.org/10.1111/jabr.12093 